\title{
Dynamic Processes During Accretion into a Black Hole
}

\author{
G.S. BISNOVATYI-KOGAN* \\ Space Research Institute, Profsoyuznaya 84/32, Moscow 117810, Russia
}

(Revised 3 January 2001)

\begin{abstract}
Accretion disc theory was first developed as a theory with the local heat balance, where the whole energy produced by a viscous heating was emitted to the sides of the disc. One of the most important new invention of this theory was a phenomenological treatment of the turbulent viscosity, known as "alpha" prescription, when the $(r \phi)$ component of the stress tensor was approximated by $(\alpha P)$ with a unknown constant $\alpha$. This prescription played the role in the accretion disc theory as well important as the mixing-length theory of convection for stellar evolution. Sources of turbulence in the accretion disc are discussed, including nonlinear hydrodynamic turbulence, convection and magnetic filed role. In parallel to the optically thick geometrically thin accretion disc models, a new branch of the optically thin accretion disc models was discovered, with a larger thickness for the same total luminosity. The choice between these solutions should be done of the base of stability analysis. The ideas underlying the necessity to include advection into the accretion disc theory are presented and first models with advection are reviewed. The present status of the solution for a low-luminous optically thin accretion disc model with advection is discussed and the limits for an advection dominated accretion flows (ADAF) imposed by the presence of magnetic field are analyzed.
\end{abstract}

\section{INTRODUCTION}

Accretion is served as a source of energy in many astrophysical objects, including different types of binary stars, binary X-ray sources, most probably quasars and active galactic nuclei (AGN). While first development of accretion theory started long time ago (Bondi and Hoyle, 1944; Bondi, 1952), the intensive development of this theory began after discovery of first X-ray sources (Giacconi et al., 1962) and quasars
(Schmidt, 1963). Accretion into stars, including neutron stars, is ended by a collision with an inner boundary, which may be a stellar surface, or outer boundary of a magnetosphere for strongly magnetized stars. We may be sure in this case, that all gravitational energy of the falling matter will be transformed into heat and radiated outward.

Situation is quite different for sources containing black holes, which are discovered in some binary $\mathrm{X}$-ray sources in the galaxy, as well as in many AGN.

*. E-mail: gkogan@mx.iki.rssi.ru 
Here matter is falling to the horizon, from where no radiation arrives and so all luminosity is formed on the way to it. The efficiency of accretion is not known from the beginning, contrary to the accretion into a star, and depends strongly on such factors, like angular momentum of the falling matter, and magnetic field embedded into it. It was first shown by Schwartsman (1971), that during spherical accretion of nonmagnetized gas the efficiency may be as small as $10^{-8}$ for sufficiently low mass fluxes. He had shown that presence of magnetic field in the accretion flux matter increase the efficiency up to about $10 \%$, and account of heating of matter due to magnetic field annihilation in the flux rises the efficiency up to about 30\% (Bisnovatyi-Kogan and Ruzmaikin, 1974). In the case of a thin disc accretion when matter has large angular momentum, the efficiency is about $1 / 2$ of the efficiency of accretion into a star with a radius equal to the radius of the last stable orbit. Matter cannot emit all the gravitational energy, part of which is absorbed by the black hole. In the case of geometrically thick and optically thin accretion discs the situation is approaching the case of spherical symmetry, and a presence of a magnetic field plays also a critical role.

Here we consider a development of the theory of a disk accretion, starting from creation of a so called "standard model", and discuss recent trends, connected with a presence of advection. Recent flow of papers (see, e.g. Narayan and Yu, 1995; Menon et al., 1997; Narayan et al., 1997a,b) considering advection dominated accretion flow (ADAF) as a solution for many astrophysical problems should be treated with some caution, because of its vague physical background. The suggestions underlying ADAF: ignorance of the magnetic field annihilation in heating of accretion plasma flow, and electron heating ONLY due to binary collisions with protons (ions) were critically analyzed in several recent papers (Blackman, 1997; Quataert, 1997; Bisnovatyi-Kogan and Lovelace, 1997; 2000; Bisnovatyi-Kogan, 1999) and the above mentioned suggestions have been strongly doubted. There are contradictions between ADAF model of accretion and observational data in radioemission for elliptical galaxies (Di Matteo et al.,
1999), and X-ray emission of Seyfert galaxy NGC4258 (Cannizzo et al., 1998, see also Chen et al., 1997).

It is, of course, dangerous to justify any physical model by astronomical observations without a firm physical ground. Here we analyze physical processes in an optically thin accretion flow at low accretion rates, connected with a presence of a small-scale magnetic field. We show, that accurate account of these processes strongly restrict the boundaries of the ADAF solution. Namely, the efficiency of the accretion flow cannot become less then about $1 / 3$ of the standard accretion disc value. It makes senseless the attempts to connect ADAF models with existence of the event horizon of a black hole, or to explain why we do not see a large luminosity from supermassive black holes in the nearby galactic nuclei.

\section{DEVELOPMENT OF THE STANDARD MODEL}

Matter falling into a black hole is gathered into a disc when its angular momentum is sufficiently high. It happens when the matter falling into a black hole comes from the neighboring ordinary star companion in the binary, or when the matter appears as a result of a tidal disruption of the star whose trajectory of motion approaches sufficiently close to the black hole, so that forces of self-gravity could be overcome. The first situation is observed in many galactic X-ray sources containing a stellar mass black hole (Cherepashchuk, 1996). A tidal disruption happens in quasars and AGN, if the model of supermassive black hole surrounded by a dense stellar cluster (Lynden-Bell, 1969) is true for these objects.

The models of the accretion disc structure around a black hole had been investigated by Lynden-Bell (1969); Pringle and Rees (1972). The modern "standard" theory of the disc accretion was formulated by Shakura (1972); Novikov and Thorne (1973); Shakura and Sunyaev (1973). It is important to note, that all authors of the accretion disc theory from USSR were students. (N.I. Shakura) or collaborators (I.D. Novikov and R.A. Sunyaev) of 
academician Ya.B. Zeldovich, who was not among the authors, but whose influence on them hardly could be overestimated.

The equations of the standard disc accretion theory were first formulated by Shakura (1972); Some corrections and generalization to general relativity (GR) were done by Novikov and Thorne (1973), see also correction to their equations in GR made by Riffert and Herold (1995). The main idea of this theory is to describe a geometrically thin non-self-gravitating disc of the mass $M_{\mathrm{d}}$, which is much smaller then the mass of the black hole $M$, by hydrodynamic equations averaged over the disc thickness $2 h$.

\subsection{Equilibrium Equations}

The small thickness of the disc in comparison with its radium $h \ll r$ indicate to small importance of the pressure gradient $\nabla P$ in comparison with gravity and inertia forces. That leads to a simple radial equilibrium equation denoting the balance between the last two forces occurring when the angular velocity of the disc $\Omega$ is equal to the Keplerian one $\Omega_{\mathrm{K}}$,

$$
\Omega=\Omega_{\mathrm{K}}=\left(\frac{G M}{r^{3}}\right)^{1 / 2} .
$$

Note, just before a last stable orbit around a black hole, and of course inside it, this suggestion fails, but in the "standard" accretion disc model the relation Eq. (1) is suggested to be fulfilled all over the disc, with an inner boundary at the last stable orbit.

The equilibrium equation in the vertical $z$-direction is determined by a balance between the gravitational force and pressure gradient

$$
\frac{\mathrm{d} P}{\mathrm{~d} z}=-\rho \frac{G M z}{r^{3}}
$$

For a thin disc this differential equation is substituted by an algebraic one, determining the half-thickness of the disc in the form

$$
h \approx \frac{1}{\Omega_{\mathrm{K}}}\left(2 \frac{P}{\rho}\right)^{1 / 2}
$$

The balance of angular momentum, related to the $\phi$ component of the Euler equation has an integral in a stationary case written as

$$
\dot{M}\left(j-j_{\text {in }}\right)=-2 \pi r^{2} 2 h t_{r \phi}, \quad t_{r \phi}=\eta r \frac{\mathrm{d} \Omega}{\mathrm{d} r} .
$$

Here $j=v_{\phi} r=\Omega r^{2}$ is a specific angular momentum, $t_{r \phi}$ is a component of the viscous stress tensor, $M>0$ is a mass flux per unit time into a black hole, $j_{0}$ is an integration constant having, after multiplication by $\dot{M}$, a physical sense of difference between viscous and advective flux of the angular momentum, when $j_{\text {in }}$ itself is equal to the specific angular momentum of matter falling into a black hole. In the standard theory the value of $j_{\text {in }}$ is determined separately, from physical considerations. For the accretion into a black hole it is suggested, that on the last stable orbit the gradient of the angular velocity is zero, corresponding to zero viscous momentum flux. In that case

$$
j_{\text {in }}=\Omega_{\mathrm{K}} r_{\text {in }}^{2},
$$

corresponding to the Keplerian angular momentum of the matter on the last stable orbit. During accretion into a slowly rotating star whose angular velocity is smaller than a Keplerian velocity on the inner edge of the disc, there is a maximum of the angular velocity close to its surface, where viscous flux is zero, and there is a boundary layer between this point and stellar surface. In that case Eq. (5) remains to be valid. The situation is different for accretion discs around rapidly rotating stars with a critical Keplerian speed on the equator. Here there is no extremum of the angular velocity of the disc, which smoothly joins the star. In stationary self-consistent situation when the accreting star remains to rotate critically during the process of a disc accretion, the specific angular momentum of matter joining the star is determined by a relation (Bisnovatyi-Kogan, 1993)

$$
j_{\text {in }}=\left.\frac{\mathrm{d} J}{\mathrm{~d} M}\right|_{\text {crit }},
$$

where the derivative is taken along the states of the star having a Keplerian equatorial speed. For stars with a polytropic structure, corresponding to equation of state 
$P=K \rho^{1+(1 / n)}$, this derivative is calculated numerically giving the value $j_{\text {in }}=0.176 \Omega_{\mathrm{K}} r_{\text {in }}^{2}$ for $n=1.5 ; 0$ for $n=2.5$; and negative values of $j_{\text {in }}$ for larger $n$.

Note, that in the pioneering paper of Shakura (1972) the integration constant $j_{\text {in }}$ was found as in Eq. (5), but was taken zero in his subsequent formulae. Importance of using $j_{\text {in }}$ in the form of Eq. (5) was noticed by Novikov and Thorne (1973), and became a feature of the standard model.

\subsection{Viscosity}

The choice of the viscosity coefficient is the most difficult and speculative problem of the accretion disc theory. In the laminar case of microscopic (atomic or plasma) viscosity, which is very low, the stationary accretion disc must be very massive and very thick, and before its formation the matter is collected by disc leading to a small flux inside. It contradicts to observations of X-ray binaries, where a considerable matter flux along the accretion disc may be explained only when viscosity coefficient is much larger then the microscopic one. It was suggested by Shakura (1972), that matter in the disc is turbulent, what determines a turbulent viscous stress tensor, parameterized by a pressure

$$
t_{r \phi}=-\alpha \rho v_{\mathrm{s}}^{2}=-\alpha P,
$$

where $v_{\mathrm{s}}$ is a sound speed in the matter. This simple presentation comes out from a relation for a turbulent viscosity coefficient $\eta_{\mathrm{t}} \approx \rho v_{\mathrm{t}} l$ with an average turbulent velocity $v_{t}$ and mean free path of the turbulent element $l$. It follows from the definition of $t_{r \phi}$ in Eq. (4), when we take $l \approx h$ from Eq. (3)

$$
t_{r \phi}=\rho v_{\mathrm{t}} h r \frac{\mathrm{d} \Omega}{\mathrm{d} r} \approx \rho v_{\mathrm{t}} v_{\mathrm{s}}=-\alpha \rho v_{\mathrm{s}}^{2},
$$

where a coefficient $\alpha<1$ is connecting the turbulent and sound speeds $v_{\mathrm{t}}=\alpha v_{\mathrm{s}}$. Presentations of $t_{r \phi}$ in Eqs. (7) and (8) are equivalent, and only when the angular velocity differs considerably from the Keplerian one the first relation to the right in Eq. (8) is more preferable. That does not appear (by definition) in the standard theory, but may happen when advective terms are included.

Development of a turbulence in the accretion disc cannot be justified simply, because a Keplerian disc is stable in linear approximation to the development of radial perturbations. It was suggested by Ya.B. Zeldovich, that in presence of very large Reynolds number $R e=(\rho v l / \eta)$ the amplitude of perturbations at which nonlinear effects become important is very low, so in this situation a turbulence may developed due to nonlinear instability even when the disc is stable in linear approximation. Another source of viscous stresses may arise from a magnetic field, but it was suggested by Shakura (1972), that magnetic stresses cannot exceed the turbulent ones.

Magnetic plasma instability as a source of the turbulence in the accretion discs has been studied extensively in last years (see review Balbus and Hawley, 1998). They used an instability of the uniform magnetic field parallel to the axis in differential rotating disc, discovered by Velikhov (1959). It could be really important in absence of any other source of the turbulence, but it is hard to believe that there is no radial or azimuthal component of the magnetic field in matter flowing into the accretion disc from the companion star. In that case the filed amplification due to twisting by a differential rotation take place without necessity of any kind of instability.

It was shown by Bisnovatyi-Kogan and Blinnikov (1976); Bisnovatyi-Kogan and Blinnikov (1977), that inner regions of a highly luminous accretion discs where pressure is dominated by radiation, are unstable to vertical convection. Development of this convection produces a turbulence, needed for a high viscosity. Other regions of a standard accretion disc should be stable to development of a vertical convection, so other ways of a turbulence excitation are needed there. With alpha- prescription of viscosity the equation of angular momentum conservation is written in the plane of the disc as

$$
\dot{M}\left(j-j_{\text {in }}\right)=4 \pi r^{2} \alpha P_{0} h .
$$

When angular velocity is far from Keplerian the relation Eq. (4) is valid with a coefficient of a 
turbulent viscosity

$$
\eta=\alpha \rho_{0} v_{\mathrm{s} 0} h
$$

where values with the index " 0 " denote the plane of the disc.

\subsection{Heat Balance}

In the standard theory a heat balance is local, what means that all heat produced by viscosity in the ring between $r$ and $r+\mathrm{d} r$ is radiated through the sides of disc at the same $r$. The heat production rate $Q_{+}$related to the surface unit of the disc is written as

$$
Q_{+}=h t_{r \phi} r \frac{\mathrm{d} \Omega}{\mathrm{d} r}=\frac{3}{8 \pi} \dot{M} \frac{G M}{r^{3}}\left(1-\frac{j_{\text {in }}}{j}\right) .
$$

Heat losses by a disc depend on its optical depth. The first standard disc model (Shakura, 1972) considered a geometrically thin disc as an optically thick in a vertical direction. That implies energy losses $Q_{\text {- from }}$ the disc due to a radiative conductivity, after a substitution of the differential equation of a heat transfer by an algebraic relation

$$
Q_{-} \approx \frac{4 a c T^{4}}{3} \frac{}{\kappa \Sigma}
$$

Here $a$ is a constant of a radiation energy density, $c$ is a speed of light, $T$ is a temperature in the disc plane $\kappa$ is a matter opacity, and a surface density

$$
\Sigma=2 \rho h
$$

here and below $\rho, T, P$ without the index " 0 " are related to the disc plane. The heat balance equation is represented by a relation

$$
Q_{+}=Q_{-}
$$

A continuity equation in the standard model of the stationary accretion flow is used for finding of a radial velocity $v_{\mathrm{r}}$

$$
v_{\mathrm{r}}=\frac{\dot{M}}{4 \pi r h \rho}=\frac{\dot{M}}{2 \pi r \Sigma} .
$$

Eqs. (1), (3), (9), (13), (14), completed by an equation of state $P(\rho, T)$ and relation for the opacity $\kappa=$ $\kappa(\rho, T)$ represent a full set of equations for a standard disc model. For power low equations of state of an ideal gas $P=P_{\mathrm{g}}=\rho R T$ ( $R$ is a gas constant), or radiation pressure $P=P_{\mathrm{r}}=\left(a T^{4} / 3\right)$, and opacity in the form of electron scattering $\kappa_{\mathrm{e}}$, or Karammers formulae $\kappa_{\mathrm{k}}$, the solution of a standard disc accretion theory is obtained analytically (Novikov and Thorne, 1973; Shakura, 1972; Shakura and Sunyaev, 1973). Checking the suggestion of a large optical thickness confirms a self-consistency of the model. One of the shortcoming of the analytical solutions of the standard model lay in the fact, that solutions for different regions of the disc with different equation of states and opacities are not matched to each other.

\subsection{Optically Thin Solution}

A few years after appearance of the standard model it was found that in addition to the optically thick disc solution there is another branch of the solution for the disc structure with the same input parameters $M, \dot{M}, \alpha$ which is also self-consistent and has a small optical thickness (Shapiro et al., 1976). Suggestion of the small optical thickness implies another equation of energy losses, determined by a volume emission

$$
Q_{-} \approx q \rho h
$$

where due to the Kirchgoff law the emissivity of the unit of a volume $q$ is connected with a Plankian averaged opacity $\kappa_{\mathrm{p}}$ by an approximate relation $q \approx$ $a c T_{0}^{4} \kappa_{\mathrm{p}}$. Note that Krammers formulae for opacity are obtained after Rosseland averaging of the frequency dependent absorption coefficient. In the optically thin limit the pressure is determined by a gas $P=P_{\mathrm{g}}$. Analytical solutions are obtained here as well, from the same equations with volume losses and gas pressure. In the optically thin solution the thickness of the disc is larger then in the optically thick one, and density is lower.

While heating via viscosity is determined mainly by heavy ions, and cooling is determined by electrons, the rate of the energy exchange between them is 
important for a thermal structure of the disc. The energy balance equations are written separately for ions and electrons. For small accretion rates and lower matter density the rate of energy exchange due to binary collisions is so slow, that in the thermal balance the ions are much hotter than the electrons. That also implies a high disc thickness and brings the standard accretion theory to the border of its applicability. Nevertheless, in the highly turbulent plasma the energy exchange between ions and electrons may be strongly enhanced due to presence of fluctuating electrical fields, where electrons and ions gain the same energy. In such conditions difference of temperatures between ions and electrons may be negligible. Regretfully, the theory of relaxation in the turbulent plasma is not completed, but there are indications to a large enhancement of the relaxation in presence of plasma turbulence, in comparison with the binary collisions (Quataert, 1997).

\section{ACCRETION DISC STRUCTURE FROM EQUATIONS DESCRIBING CONTINUOUSLY OPTICALLY THIN AND OPTICALLY THICK DISC REGIONS}

In order to find equations of the disc structure valid in both limiting cases of optically thick and optically thin disc, and smoothly describing transition between them, use Eddington approximation for obtaining formulae for a heat flux and for a radiation pressure (Artemova et al. 1996). Suppose that disc is geometrically thin and has a constant density along $z$ - axis. Defining $S_{\mathrm{r}}$ as the energy density of the radiation. $F_{\text {rad }}$ as radiation flux in $z$-direction, $P_{\text {rad }}$ as radiation pressure we write momentum equations for averaged $\kappa_{\mathrm{p}}$ in the form (Bisnovatyi-Kogan, 1989)

$$
\begin{gathered}
\frac{\mathrm{d} F_{\mathrm{rad}}}{\mathrm{d} z}=-\rho c \kappa_{\mathrm{p}} a T^{4}\left(\frac{S_{\mathrm{r}}}{a T^{4}}-1\right), \\
c \frac{\mathrm{d} P_{\mathrm{rad}}}{\mathrm{d} z}=-\kappa_{\mathrm{e}} \rho F_{\mathrm{rad}} .
\end{gathered}
$$

Consider the case when scattering opacity $\kappa_{\mathrm{e}}$ is much larger then absorption opacity $\kappa_{\mathrm{p}}$, and suggest that heat production rate is proportional to the mass density $\rho$. Then, neglecting the flux in the radial direction we get

$$
F_{\text {rad }}=2 \frac{F_{0}}{\Sigma_{0}} \rho z
$$

where $F_{0}$ is the flux from the unit surface of the disc at $z=h$. Substituting Eq. (19) into Eq. (17) we get

$$
S_{\mathrm{r}}=3 P_{\mathrm{rad}}=a T^{4}\left(1-\frac{2 F_{0}}{c \kappa_{\mathrm{p}} a T^{4} \Sigma_{0}}\right)
$$

Using Eq. (19) in Eq. (18) we get

$$
c \frac{\mathrm{d} P_{\mathrm{rad}}}{\mathrm{d} z}=-2 \kappa_{\mathrm{e}} \frac{F_{0}}{\Sigma_{0}} \rho^{2} z .
$$

Introducing the scattering optical depth

$$
\tau=\int_{z}^{\infty} \kappa_{\mathrm{e}} \rho \mathrm{d} z=\kappa_{\mathrm{e}} \rho(h-z)=\tau_{0}-\kappa_{\mathrm{e}} \rho z
$$

$$
\tau_{0}=\kappa_{\mathrm{e}} \rho h=\frac{1}{2} \kappa_{\mathrm{e}} \Sigma_{0}
$$

we rewrite Eq. (21) in the form

$$
c \frac{\mathrm{d} P_{\mathrm{rad}}}{\mathrm{d} \tau}=2 \frac{F_{0}}{\Sigma_{0}} \frac{\tau_{0}-\tau}{\kappa_{\mathrm{e}}} .
$$

Solve Eq. (23) with the following boundary condition

$$
\left.F_{\mathrm{rad}}\right|_{\tau=0}=F_{0}=\frac{\left.c S_{\mathrm{r}}\right|_{\tau=0}}{2}=\frac{\left.3 c P_{\mathrm{rad}}\right|_{\tau=0}}{2},
$$

resulting in

$$
c P_{\mathrm{rad}}=F_{0}\left(\frac{2}{3}+\tau-\frac{\tau^{2}}{2 \tau_{0}}\right) .
$$

In the symmetry plane of the disc at $\tau=\tau_{0}$ we have

$$
c P_{\mathrm{rad}, 0}=F_{0}\left(\frac{2}{3}+\frac{\tau_{0}}{2}\right) .
$$

Using Eq. (26) in Eq. (20) we get in the symmetry 
plane where $T=T_{0}$

$$
F_{0}=c a T_{0}^{4}\left(2+\frac{3 \tau_{0}}{2}+\frac{1}{\tau_{\alpha 0}}\right)^{-1}
$$

where absorption optical depth

$$
\begin{gathered}
\tau_{\alpha}=\int_{z}^{\infty} \kappa_{\mathrm{p}} \rho \mathrm{d} z=\kappa_{\mathrm{p}} \rho(h-z)=\tau_{\alpha 0}-\kappa_{\mathrm{p}} \rho z, \\
\tau_{\alpha 0}=\kappa_{\mathrm{p}} \rho h=\frac{1}{2} \kappa_{\mathrm{p}} \Sigma_{0} .
\end{gathered}
$$

Introducing effective optical depth

$$
\tau_{*}=\left(\tau_{0} \tau_{\alpha 0}\right)^{1 / 2},
$$

we get finally the expressions for the vertical energy flux from the disc $F_{0}$ and the radiation pressure in the symmetry plane as

$$
\begin{gathered}
F_{0}=\frac{2 a c T_{0}^{4}}{3 \tau_{0}}\left(1+\frac{4}{3 \tau_{0}}+\frac{2}{3 \tau_{*}^{2}}\right)^{-1}, \\
P_{\mathrm{rad}, 0}=\frac{a T_{0}^{4}}{3} \frac{1+\left(4 / 3 \tau_{0}\right)}{1+\left(4 / 3 \tau_{0}\right)+\left(2 / 3 \tau_{*}^{2}\right)} .
\end{gathered}
$$

At $\tau_{0} \gg \tau_{*} \gg 1$ we have Eq. (12) from Eq. (30). In the optically thin limit $\tau_{*} \ll \tau_{0} \ll 1$ we get

$$
F_{0}=a c T_{0}^{4} \tau_{\alpha 0}, \quad P_{\text {rad }, 0}=\frac{2}{3} a c T_{0}^{4} \tau_{\alpha 0} .
$$

Using $F_{0}$ instead of $Q_{-}$and equation of state $P=$ $\rho R T+P_{\text {rad }, 0}$, the equations of accretion disc structure together with equation

$$
Q_{+}=F_{0}
$$

with $Q_{+}$from Eq. (11), have been solved numerically by Artemova et al. (1996). It occurs that two solutions, optically thick and optically thin, exist separately when luminosity is not very large. Two solutions intersect at $\dot{m}=\dot{m}_{\mathrm{b}}$ and there is no global solution for accretion disc at $\dot{m}>\dot{m}_{\mathrm{b}}$ (see Fig. 1). It was concluded by Artemova et al. (1996), that in order to obtain a global physically

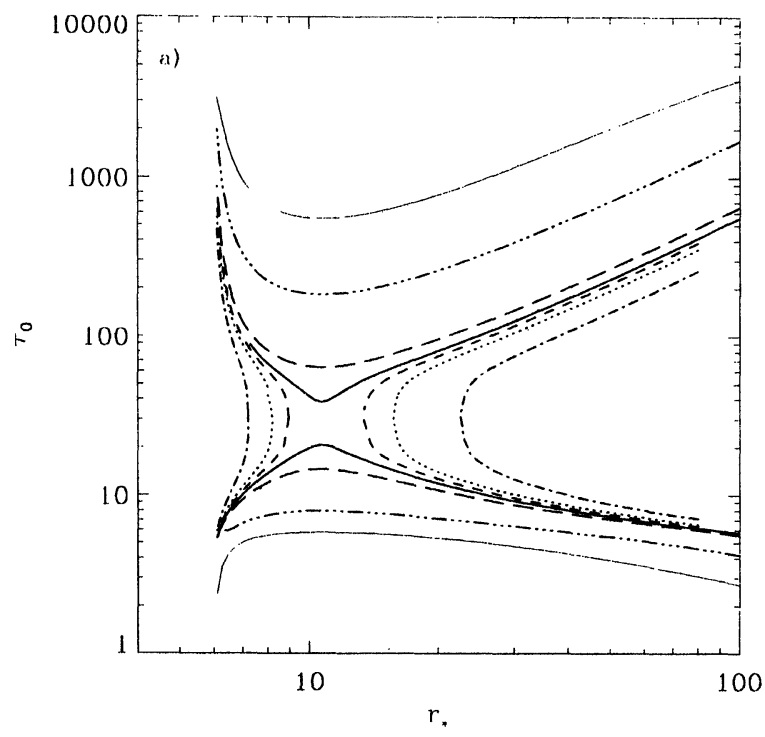

FIGURE 1 The dependencies of the optical depth $\tau_{0}$ on radius, $\tau_{*}=r / r_{\mathrm{g}}$, for the case $M_{\mathrm{BH}}=10^{8} M_{\odot}, \alpha=1.0$ and different values of $\dot{m}$. The thins solid, dot-triple dash, long dashed, heavy solid, short dashed, dotted and dot-dashed curves correspond to $\dot{m}=1.0,3.0,8.0,9.35,10.0,11.0,15.0$, respectively. The upper curves correspond to the optically thick family, lower curves correspond to the optically thin family.

meaningful solution at $\dot{m}>\dot{m}_{\mathrm{b}}$, account for an advective term in Eq. (33) is needed.

\section{ACCRETION DISCS WITH ADVECTION}

Standard model gives somewhat nonphysical behavior near the inner edge of the accretion disc around a black hole. For high mass fluxes when central regions are radiation-dominated $\left(P \approx P_{\mathrm{r}}, \kappa \approx \kappa_{\mathrm{e}}\right)$, the radial dependence follows relations (Shakura and Sunyaev, 1973)

$$
\begin{aligned}
& \rho \sim r^{3 / 2} \mathscr{J}^{-2} \rightarrow \infty, \quad T \sim r^{-3 / 8}, \\
& h \sim \mathscr{J} \rightarrow 0, \quad \Sigma \sim r^{3 / 2} \mathscr{J}^{-1} \rightarrow \infty, \\
& v_{\mathrm{r}} \sim r^{-5 / 2} \mathscr{J} \rightarrow 0,
\end{aligned}
$$

where limits relate to the inner edge of the disc with 
$r=r_{\text {in }}$

$$
\mathscr{J}=1-\frac{j_{\text {in }}}{j}=1-\sqrt{\frac{r_{\text {in }}}{r}} .
$$

At smaller $\dot{M}$, when near the inner edge $P \approx P_{\mathrm{g}}, \kappa \approx$ $\kappa_{\mathrm{e}}$, there are different type of singularities

$$
\begin{gathered}
\rho \sim r^{-33 / 20} \mathscr{J}^{2 / 5} \rightarrow 0, \quad T \sim r^{-9 / 10} \mathscr{J}^{2 / 5} \rightarrow 0, \\
h \sim r^{21 / 20} \mathscr{J}^{1 / 5} \rightarrow 0, \quad \Sigma \sim r^{-3 / 5} \mathscr{J}^{3 / 5} \rightarrow 0, \\
v_{\mathrm{r}} \sim r^{-2 / 5} \mathscr{J}^{-3 / 5} \rightarrow \infty .
\end{gathered}
$$

This results from the local form of the equations of the thermal balance (Eq. (14)). It is clear from physical ground, that when a local heat production due to viscosity goes to zero, the heat brought by radial motion of matter along the accretion disc becomes more important. In presence of this advective heating (or cooling term, depending on the radial entropy $S$ gradient) written as

$$
Q_{\mathrm{adv}}=\frac{\dot{M}}{2 \pi r} T \frac{\mathrm{d} S}{\mathrm{~d} r},
$$

the equation of a heat balance is modified to

$$
Q_{+}+Q_{\mathrm{adv}}=Q_{-} .
$$

In order to describe self-consistently the structure of the accretion disc we should also modify the radial disc equilibrium, including pressure and inertia terms

$$
r\left(\Omega^{2}-\Omega_{\mathrm{K}}^{2}\right)=\frac{1}{\rho} \frac{\mathrm{d} P}{\mathrm{~d} r}-v_{\mathrm{r}} \frac{\mathrm{d} v_{\mathrm{r}}}{\mathrm{d} r}
$$

Appearance of inertia term leads to transonic radial flow with a singular point. Conditions of a continuous passing of the solution through a critical point choose a unique value of the integration constant $j_{\text {in }}$. First approximate solution for the advective disc structure have been obtained by Paczyńsci and BisnovatyiKogan (1981), but a corresponding set of equations had been discussed earlier (Hõshi and Shibazaki, 1977; Liang and Thompson, 1980). Attempts to find a solution for advective disc structure gave the following results (Abramovicz et al., 1988; Matsumoto et al., 1984). For moderate values of $\dot{M}$ a unique continuous transonic solution was found, passing through singular points, and corresponding to a unique value of $j_{\text {in }}$. The number of critical point in the radial flow could be more then unity. This is connected with the gravitational potential $\phi_{\mathrm{g}}$ in papers dealing with advective disc solutions was different from Newtonian one (Paczyńsci and Wiita, 1980)

$$
\phi_{\mathrm{g}}=\frac{G M}{r-r_{\mathrm{g}}}, \quad r_{\mathrm{g}}=\frac{2 G M}{c^{2}} .
$$

The advantage of this potential is a realistic approximation of the general relativistic (GR) effects, namely, infinitive gravitational attraction at a gravitational radius $r_{\mathrm{g}}$, and existence of the stable circular orbits only up to $r=3 r_{\mathrm{g}}$, like in exact GR. Appearance of two critical points for a radial flow in this potential was analyzed by Chakrabarti and Molteni (1993). The second reason of multiplicity of singular points is connected with using of equations averaged over a thickness of the disc. That changes a structure of hydrodynamic equations, leading to a position of singular points not coinciding with a unit Mach number point, and increasing a number of critical points.

When $\dot{M}$ is becoming so high, that radiation pressure starts to be important, problems appear in a construction of the advective disc model connected with loss of uniqueness of the transonic solution. So, with increasing of $\dot{M}$ the solution becomes nonunique at some parameters, or was not found at all (Abramovicz et al., 1988; Matsumoto et al., 1984). At high $\dot{M}$ the integral curves are sensitive to input conditions: form of viscosity stresses Eq. (4) or Eq. (7), choice of boundary conditions etc. In addition to continuous solutions with standing shock waves have been investigated (Chakrabarti, 1996).

\section{MAGNETIC FIELD ENHANCEMENT IN QUASISPHERICAL ACCRETION}

A matter flowing into a black hole from a companion star or from interstellar medium is magnetized. Due to 
more rapid increase of a magnetic energy in comparison with any other kind of energy, the dynamical and any other role of the magnetic field is becoming more and more important when matter flows inside. It was first indicated by Schwartsman (1971) the exceptional importance of the magnetic field for an energy release during accretion into a black hole. It was shown, that due to more rapid growth of magnetic energy its density $E_{\mathrm{M}}$ approaches a density of a kinetic energy $E_{\mathrm{k}}$, and proposed a hypothesis of equipartition $E_{\mathrm{M}} \approx E_{\mathrm{k}}$, supported by continuous annihilation of the magnetic field, for the smaller radii of the flow where the main energy production happens. This hypothesis is usually accepted in the modern picture of accretion (see e.g Narayan and $\mathrm{Yu}$, 1995). Schwartsman (1971) considered an averaged quasistationary picture with local equipartition, another variant of magnetic accretion, where equipartition and magnetic field annihilation is accompanied by formation of shock waves was considered by Bisnovatyi-Kogan and Sunyaev (1972), see also Chang and Ostriker (1985). More accurate account of the heating of matter by magnetic field annihilation was done by BisnovatyiKogan and Ruzmaikin (1974), where exact nonstationary solution for a field amplification in the radial accretion flow was also obtained.

Let us consider a more general problem of the field amplification in the accretion flow where gas has an angular momentum and field annihilation is approximated by a finite "turbulent" conductivity. This approach should reproduce a smooth averaged flow, considered by Schwartsman. In the known stationary velocity field $\mathbf{v}(r, \theta)$ the magnetic field $\mathbf{B}$ is described by Maxwell equations in MHD approximation (without displacement currents), with a finite conductivity

$$
\begin{aligned}
\nabla \times \mathbf{B} & =\frac{4 \pi}{c} \mathbf{j}, \quad \nabla \times \mathbf{E}=-\frac{1}{c} \frac{\partial \mathbf{B}}{\partial t}, \quad \nabla \cdot \mathbf{B}=0, \\
\mathbf{j} & =\sigma\left(\mathbf{E}+\frac{1}{c} \mathbf{v} \times \mathbf{B}\right),
\end{aligned}
$$

where $\sigma$ is the plasma conductivity, $\mathbf{j}$ is the electrical current density, and $\mathbf{E}$ is the electrical field strength. Consider an axisymmetrical picture in a spherical coordinate system $r, \theta, \phi$ where all $\phi$ derivatives are zero, but all vectors $\mathbf{v}, \mathbf{B}, \mathbf{E}, \mathbf{j}$ may have nonzero all three components. The equation containing only magnetic field, following from Eq. (41)

$$
\frac{\partial \mathbf{B}}{\partial t}=\nabla \times[\mathbf{v} \times \mathbf{B}]+\frac{c^{2}}{4 \pi \sigma} \Delta \mathbf{B},
$$

in spherical coordinates for axisymmetrical case are written as

$$
\begin{aligned}
\frac{\partial B_{\mathrm{r}}}{\partial t}= & \frac{1}{r \sin \theta} \frac{\partial}{\partial \theta}\left[\sin \theta\left(v_{\mathrm{r}} B_{\theta}-v_{\theta} B_{\mathrm{r}}\right)\right]+\frac{c^{2}}{4 \pi \sigma} \\
\times & {\left[\frac{1}{r^{2}} \frac{\partial}{\partial r}\left(r^{2} \frac{\partial B_{\mathrm{r}}}{\partial r}\right)+\frac{1}{r^{2} \sin \theta} \frac{\partial}{\partial \theta}\left(\sin \theta \frac{\partial B_{\mathrm{r}}}{\partial \theta}\right)\right.} \\
- & \left.\frac{2 B_{\mathrm{r}}}{r^{2}}-\frac{2}{r^{2} \sin \theta} \frac{\partial}{\partial \theta}\left(\sin \theta B_{\theta}\right)\right] \\
\frac{\partial B_{\theta}}{\partial t}= & -\frac{1}{r} \frac{\partial}{\partial r}\left[r\left(v_{\mathrm{r}} B_{\theta}-v_{\theta} B_{\mathrm{r}}\right)\right]+\frac{c^{2}}{4 \pi \sigma} \\
& \times\left[\frac{1}{r^{2}} \frac{\partial}{\partial r}\left(r^{2} \frac{\partial B_{\theta}}{\partial r}\right)+\frac{1}{r^{2} \sin \theta} \frac{\partial}{\partial \theta}\right. \\
& \left.\times\left(\sin \theta \frac{\partial B_{\theta}}{\partial \theta}\right)-\frac{B_{\theta}}{r^{2} \sin ^{2} \theta}+\frac{2}{r^{2}} \frac{\partial B_{\mathrm{r}}}{\partial \theta}\right], \\
\frac{\partial B_{\phi}}{\partial t}= & \left.\frac{1}{r} \frac{\partial}{\partial r}\left[r\left(v_{\phi} B_{\mathrm{r}}-v_{\mathrm{r}} B_{\phi}\right)\right]-\frac{\partial}{\partial \theta}\left[v_{\theta} B_{\phi}-v_{\phi} B_{\theta}\right)\right] \\
+ & \frac{c^{2}}{4 \pi \sigma}\left[\frac{1}{r^{2}} \frac{\partial}{\partial r}\left(r^{2} \frac{\partial B_{\phi}}{\partial r}\right)\right. \\
+ & \left.\frac{1}{r^{2} \sin \theta} \frac{\partial}{\partial \theta}\left(\sin \theta \frac{\partial B_{\phi}}{\partial \theta}\right)-\frac{B_{\phi}}{r^{2} \sin ^{2} \theta}\right]
\end{aligned}
$$

These equations are completed by the condition of zero divergency of the magnetic field, which for the chosen case has a view

$$
\frac{1}{r^{2}} \frac{\partial}{\partial r}\left(r^{2} B_{\mathrm{r}}\right)+\frac{1}{r \sin \theta} \frac{\partial}{\partial \theta}\left(\sin \theta B_{\theta}\right)=0 .
$$




\subsection{Amplification of the Magnetic Field at a Spherical Accretion}

For a spherical accretion with $\mathbf{v}=\left(v_{\mathrm{r}}, 0,0\right)$ the Eqs. (43)-(45) reduce to

$$
\begin{aligned}
\frac{\partial B_{\mathrm{r}}}{\partial t}= & \frac{1}{r \sin \theta} \frac{\partial}{\partial \theta}\left(\sin \theta v_{\mathrm{r}} B_{\theta}\right)+\frac{c^{2}}{4 \pi \sigma} \\
& \times\left[\frac{1}{r^{2}} \frac{\partial}{\partial r}\left(r^{2} \frac{\partial B_{\mathrm{r}}}{\partial r}\right)+\frac{1}{r^{2} \sin \theta} \frac{\partial}{\partial \theta}\left(\sin \theta \frac{\partial B_{\mathrm{r}}}{\partial \theta}\right)\right. \\
& \left.-\frac{2 B_{\mathrm{r}}}{r^{2}}-\frac{2}{r^{2} \sin \theta} \frac{\partial}{\partial \theta}\left(\sin \theta B_{\theta}\right)\right]
\end{aligned}
$$$$
\frac{\partial B_{\theta}}{\partial t}=-\frac{1}{r} \frac{\partial}{\partial r}\left(r v_{\mathrm{r}} B_{\theta}\right)+\frac{c^{2}}{4 \pi \sigma}\left[\frac{1}{r^{2}} \frac{\partial}{\partial r}\left(r^{2} \frac{\partial B_{\theta}}{\partial r}\right)\right.
$$$$
+\frac{1}{r^{2} \sin \theta} \frac{\partial}{\partial \theta}\left(\sin \theta \frac{\partial B_{\theta}}{\partial \theta}\right)
$$$$
\left.-\frac{B_{\theta}}{r^{2} \sin ^{2} \theta}+\frac{2}{r^{2}} \frac{\partial B_{\mathrm{r}}}{\partial \theta}\right]
$$$$
\frac{\partial B_{\phi}}{\partial t}=-\frac{1}{r} \frac{\partial}{\partial r}\left(r v_{\mathrm{r}} B_{\phi}\right)+\frac{c^{2}}{4 \pi \sigma}\left[\frac{1}{r^{2}} \frac{\partial}{\partial r}\left(r^{2} \frac{\partial B_{\phi}}{\partial r}\right)\right.
$$$$
\left.+\frac{1}{r^{2} \sin \theta} \frac{\partial}{\partial \theta}\left(\sin \theta \frac{\partial B_{\phi}}{\partial \theta}\right)-\frac{B_{\phi}}{r^{2} \sin ^{2} \theta}\right] \text {. }
$$

For initial nonstationary stage of the field amplification, when field dissipation is negligible, analytical solution may be obtained (BisnovatyiKegan and Ruzmaikin, 1974). From Eq. (47) with account of Eq. (45), and from Eqs. (48), (49) we get

$$
\frac{\mathrm{d}\left(r^{2} B_{\mathrm{r}}\right)}{\mathrm{d} t}=0, \quad \frac{\mathrm{d}\left(r v_{\mathrm{r}} B_{\theta}\right)}{\mathrm{d} t}=0, \quad \frac{\mathrm{d}\left(r v_{\mathrm{r}} B_{\phi}\right)}{\mathrm{d} t}=0,
$$

where the full Lagrangian derivative is determined as

$$
\frac{\mathrm{d}}{\mathrm{d} t}=\frac{\partial}{\partial t}+v_{\mathrm{r}} \frac{\partial}{\partial r} .
$$

A solution of Eqs. (50) and (51) is determined by 4 first integrals of the characteristic equations

$$
\begin{gathered}
C_{1}=t-\int \frac{\mathrm{d} r}{v_{\mathrm{r}}}, \quad C_{2}=r^{2} B_{\mathrm{r}}, \quad C_{3}=r v_{\mathrm{r}} B_{\theta}, \\
C_{4}=r v_{\mathrm{r}} B_{\phi} .
\end{gathered}
$$

For a free fall case with $v_{\mathrm{r}}=-\sqrt{(2 G M / r)}$ we get

$$
C_{1}=t+\frac{2}{3} \frac{r^{3 / 2}}{\sqrt{2 G M}}
$$

The initial condition problem at $t=0$ is solved separately for poloidal and toroidal fields. For initially uniform field $B_{\mathrm{r} 0}=B_{0} \cos \theta, \quad B_{\theta 0}=$ $-B_{0} \sin \theta$ we obtain the integrals

$$
\begin{gathered}
C_{1}=\frac{2}{3} \frac{r^{3 / 2}}{\sqrt{2 G M}}, \quad C_{2}=r^{2} B_{0} \cos \theta, \\
C_{3}=\sqrt{2 G M r} B_{0} \sin \theta .
\end{gathered}
$$

The relation between the integrals found at initial moment after excluding $r\left(C_{1}\right)$ is valid at any time, so finally we get from Eq. (52) the solution (Bisnovatyi-Kegan and Ruzmaikin, 1974)

$$
\begin{gathered}
B_{\mathrm{r}}=\frac{B_{0} \cos \theta}{r^{2}}\left(r^{3 / 2}+\frac{3}{2} t \sqrt{2 G M}\right)^{4 / 3}, \\
B_{\theta}=-\frac{B_{0} \sin \theta}{\sqrt{r}}\left(r^{3 / 2}+\frac{3}{2} t \sqrt{2 G M}\right)^{1 / 3} .
\end{gathered}
$$

The radial component of the field is growing most rapidly. It is $\sim r^{-2}$ for sufficiently large times, $\sim t^{4 / 3}$ at given sufficiently small radius, and is growing with time everywhere.

For initially dipole magnetic field $B_{\mathrm{r} 0}=$ $\left(B_{0} \cos \theta / r^{3}\right), B_{\theta 0}=-\left(B_{0} \sin \theta / 2 r^{3}\right)$ we obtain the following solution

$$
\begin{gathered}
B_{\mathrm{r}}=\frac{B_{0} \cos \theta}{r^{2}}\left(r^{3 / 2}+\frac{3}{2} t \sqrt{2 G M}\right)^{-2 / 3}, \\
B_{\theta}=-\frac{B_{0} \sin \theta}{2 \sqrt{r}}\left(r^{3 / 2}+\frac{3}{2} t \sqrt{2 G M}\right)^{-5 / 3} .
\end{gathered}
$$


Here the magnetic field is decreasing everywhere with time, tending to zero. That describes a pressing of a dipole magnetic field to a stellar surface. The azimuthal stellar magnetic field if confined inside the star. When outer layers of the star are compressing with a free-fall speed, then for initial field distribution $B \phi_{0}=B_{0} r^{n} f(\theta)$ the change of magnetic field with time is described by a relation

$$
B_{\phi}=-\frac{B_{0} f(\theta)}{\sqrt{r}}\left(r^{3 / 2}+\frac{3}{2} t \sqrt{2 G M}\right)^{1 / 3+n} .
$$

\section{STATIONARY PICTURE OF THE MAGNETIC FIELD DISTRIBUTION IN QUASI-SPHERICAL ACCRETION: TURBULENCE, EQUIPARTITION AND FIELD ANNIHILATION; TWO- TEMPERATURE ADVECTIVE DISCS}

In the optically thin accretion discs at low mass fluxes the density of the matter is low and energy exchange between electrons and ions due to binary collisions is slow. In this situation, due to different mechanisms of heating and cooling for electrons and ions, they may have different temperatures. First it was realized by Shapiro et al. (1976) where advection was not included. It was noticed by Narayan and Yu (1995) (see also Ichimaru, 1977), that advection in this case is becoming extremely important. It may carry the main energy flux into a black hole, leaving rather low efficiency of the accretion up to $10^{-3}-10^{-4}$ (ADAF). This conclusion is valid only when the effects, connected with magnetic field annihilation and heating of matter due to it are neglected.

In the ADAF solution the ion temperature is about a virial one $k T_{\mathrm{i}} \sim G M m_{\mathrm{i}} / r$, what means that even at high initial angular momentum the disc becomes very thick, forming practically a quasi-spherical accretion flow. It is connected also with an "alpha" prescription of viscosity. At high ion temperatures, connected with a strong viscous heating, the ionic pressure becomes high, making the viscosity very effective. So, due to suggestion of "alpha" viscosity in the situation, when energy losses by ions are very low, some kind of a "thermo-viscous" instability is developed, because heating increases a viscosity, and viscosity increases a heating. Development of this instability leads to formation of ADAF.

A full account of the processes, connected with a presence of magnetic field in the flow, is changing considerably the picture of ADAF. It was shown by Schwartsman (1971), that radial component of the magnetic field increases so rapidly in the spherical flow, that equipartition between magnetic and kinetic energy is reached in the flow far from the black hole horizon. In the region where the main energy production takes place, the condition of equipartition takes place. In presence of a high magnetic field the efficiency of a radiation during accretion of an interstellar matter into a black hole increase enormously from $\sim 10^{-8}$ up to $\sim 0.1$ (Schwartsman, 1971), due to efficiency of a magneto-bremstrahlung radiation. So possibility of ADAF regime for a spherical accretion was noticed long time ago. To support the condition of equipartition a continuous magnetic field reconnection is necessary, leading to annihilation of the magnetic flux and heating of matter due to Ohmic heating. It was shown by BisnovatyiKogan and Ruzmaikin (1974), that due to Ohmic heating the efficiency of a radial accretion into a black hole may become as high as $\sim 30 \%$. The rate of the Ohmic heating in the condition of equipartition was obtained in the form

$$
T \frac{\mathrm{d} S}{\mathrm{~d} r}=-\frac{3}{2} \frac{B^{2}}{8 \pi \rho r} .
$$

In the supersonic flow of the radial accretion equipartition between magnetic and kinetic energy was suggested (Schwartsman, 1971):

$$
\frac{B^{2}}{8 \pi} \approx \frac{\rho v_{\mathrm{r}}^{2}}{2}=\frac{\rho G M}{r} .
$$

For the disc accretion, where there is more time for a field dissipation, almost equpartition was suggested (Shakura, 1972) between magnetic and turbulent energy, what reduces with account of "alpha" 
prescription of viscosity to a relation

$$
\frac{B^{2}}{8 \pi} \sim \frac{\rho v_{\mathrm{t}}^{2}}{2}=\frac{3}{2} \alpha_{\mathrm{m}}^{2} P
$$

where $\alpha_{\mathrm{m}}$ characterizes a magnetic viscosity in a way similar to the turbulent $\alpha$ viscosity. It was suggested by Bisnovatyi-Kogan and Ruzmaikin (1976) the similarity between viscous and magnetic Reynolds numbers, or between turbulent and magnetic viscosity coefficients

$$
R e=\frac{\rho v l}{\eta}, \quad R e_{\mathrm{m}}=\frac{\rho v l}{\eta_{\mathrm{m}}}
$$

where the turbulent magnetic viscosity $\eta_{\mathrm{m}}$ is connected with a turbulent conductivity $\sigma$

$$
\sigma=\frac{\rho c^{2}}{4 \pi \eta_{\mathrm{m}}}
$$

Taking $\eta_{\mathrm{m}}=\left(\alpha_{\mathrm{m}} / \alpha\right) \eta$, we get a turbulent conductivity

$$
\sigma=\frac{c^{2}}{4 \pi \alpha_{\mathrm{m}} h v_{\mathrm{s}}}, \quad v_{\mathrm{s}}^{2}=\frac{P_{\mathrm{g}}}{\rho}
$$

in the optically thin discs. For the radial accretion the turbulent conductivity may contain mean free path of a turbulent element $l_{\mathrm{t}}$, and turbulent viscosity $v_{\mathrm{t}}$ in Eq. (63) instead of $h$ and $v_{\mathrm{s}}$. In ADAF solutions, where ionic temperature is of the order of the virial one the two suggestions Eqs. (59) and (60) almost coincide at $\alpha_{\mathrm{m}} \sim 1$.

The heating of the matter due to an Ohmic dissipation may be obtained from the Ohm's law for a radial accretion in the form

$$
T \frac{\mathrm{d} S}{\mathrm{~d} r}=-\frac{\sigma \varepsilon^{2}}{\rho v_{\mathrm{r}}} \approx-\sigma \frac{v_{\mathrm{t}}^{2} B^{2}}{\rho v_{\mathrm{r}} c^{2}}=-\frac{B^{2} v_{\mathrm{t}}}{\rho 4 \pi \alpha_{\mathrm{m}} v_{\mathrm{r}} l_{\mathrm{t}}}
$$

what coincides with Eq. (58) when $\alpha_{\mathrm{m}}=\left(4 r v_{\mathrm{t}} / 3 v_{\mathrm{r}} l_{\mathrm{t}}\right)$, or $l_{\mathrm{t}}=\left(4 r v_{\mathrm{t}} / 3 v_{\mathrm{r}} \alpha_{\mathrm{m}}\right)$. Here a local electrical field strength in a highly conducting plasma is of the order of $\varepsilon \sim\left(v_{\mathrm{t}} B / c\right)$ for the radial accretion.

Equations for a radial temperature dependence in the accretion disc, separate for the ions and electrons are written as

$$
\frac{\mathrm{d} E_{\mathrm{i}}}{\mathrm{d} t}-\frac{P_{\mathrm{i}}}{\rho^{2}} \frac{\mathrm{d} \rho}{\mathrm{d} t}=\mathscr{H}_{\eta \mathrm{i}}+\mathscr{H}_{\mathrm{Bi}}-Q_{\mathrm{ie}}
$$

$$
\begin{aligned}
\frac{\mathrm{d} E_{\mathrm{e}}}{\mathrm{d} t} & -\frac{P_{\mathrm{e}}}{\rho^{2}} \frac{\mathrm{d} \rho}{\mathrm{d} t}=\mathscr{H}_{\eta \mathrm{e}}+\mathscr{H}_{\mathrm{Be}} \\
& +\mathscr{Q}_{\mathrm{ie}}-\mathscr{C}_{\mathrm{brem}}-\mathscr{C}_{\mathrm{cyc}},
\end{aligned}
$$

Here $\frac{\mathrm{d}}{\mathrm{d} t}=\frac{\partial}{\partial t}+v_{\mathrm{r}} \frac{\partial}{\partial r}$. A rate of a viscous heating of ions $\mathscr{H}_{\eta \mathrm{i}}$ is obtained from Eq. (11) as

$$
\begin{gathered}
\mathscr{H}_{\eta \mathrm{i}}=\frac{2 \pi T}{\dot{M}} Q_{+}=\frac{3}{2} \alpha \frac{v_{\mathrm{K}} v_{\mathrm{s}}^{2}}{r}, \\
\mathscr{H}_{\eta \mathrm{e}} \leq \sqrt{\frac{m_{\mathrm{e}}}{m_{\mathrm{i}}}} \mathscr{H}_{\eta \mathrm{i}} .
\end{gathered}
$$

Combining Eqs. (3), (15), (9), (13), we get

$$
\begin{gathered}
v_{\mathrm{r}}=\alpha \frac{v_{\mathrm{s}}^{2}}{v_{\mathrm{K}} \mathscr{J}}, \quad h=\sqrt{2} \frac{v_{\mathrm{s}}}{v_{\mathrm{K}}} \tau, \\
\rho=\frac{\dot{M}}{4 \pi \alpha \sqrt{2}} \frac{v_{K}^{2} \mathscr{J}}{r^{2} v_{\mathrm{s}}^{3}}
\end{gathered}
$$

where $v_{\mathrm{K}}=r \Omega_{\mathrm{K}}$. The rate of the energy exchange between ions and electrons due to the binary collisions was obtained by Landau (1937) and Spitzer (1940)

$$
Q_{\mathrm{ie}} \approx \frac{4(2 \pi)^{1 / 2} n e^{4}}{m_{\mathrm{i}} m_{\mathrm{e}}}\left(\frac{T_{\mathrm{e}}}{m_{\mathrm{e}}}+\frac{T_{\mathrm{i}}}{m_{\mathrm{i}}}\right)^{\frac{-3}{2}} \ln \Lambda\left(T_{\mathrm{i}}-T_{\mathrm{e}}\right)
$$

with $\ln \Lambda=\mathcal{O}(20)$ the Coulomb logarithm. Thermodynamic functions must take into account a possible relativistic effects for electrons, which energy may exceed $m_{\mathrm{e}} c^{2}$. Neglecting pair formation for a low density accretion disc, we may write an exact expression for a pressure

$$
\begin{aligned}
P_{\mathrm{g}} & =P_{\mathrm{i}}+P_{\mathrm{e}}=n_{\mathrm{i}} k T_{\mathrm{e}}+n_{\mathrm{e}} k T_{\mathrm{p}} \\
& =n_{\mathrm{e}} k\left(T_{\mathrm{e}}+T_{\mathrm{i}}\right)
\end{aligned}
$$

and an approximate expression for an energy, containing a smooth interpolation between non- 
relativistic and relativistic electrons

$$
\begin{aligned}
E_{\mathrm{g}} & =E_{\mathrm{e}}+E_{\mathrm{i}} \\
& \approx \frac{(3 / 2) m_{\mathrm{e}} c^{2}+3 k T_{\mathrm{e}}}{m_{\mathrm{e}} c^{2}+k T_{\mathrm{e}}} \frac{k T_{\mathrm{e}}}{m_{\mathrm{p}}}+\frac{3}{2} \frac{k T_{\mathrm{i}}}{m_{\mathrm{p}}} .
\end{aligned}
$$

The Bremstrahlung $C_{\text {brem }}$ and magneto-Bremstrahlung $C_{\text {cyc }}$ cooling of Maxwellian semi-relativistic electrons, with account of free-bound radiation in nonrelativistic limit, may be written as by interpolation of limiting cases (Bisnovatyi-Kogan and Ruzmaikin, 1976)

$$
\begin{aligned}
& C_{\text {brem }} \approx \frac{2 \times 10^{30} m_{\mathrm{e}} c^{2}+3 \times 10^{32}\left(k T_{\mathrm{e}}\right)^{3 / 2}}{m_{\mathrm{e}} c^{2}+k T_{\mathrm{e}}}\left(k T_{\mathrm{e}}\right)^{1 / 2}, \\
& C_{\mathrm{cyc}} \approx \frac{1.7 \times 10^{16} m_{\mathrm{e}} c^{2}+1.7 \times 10^{22}\left(k T_{\mathrm{e}}\right)^{2}}{m_{\mathrm{e}} c^{2}+k T_{\mathrm{e}}} k T_{\mathrm{e}} B^{2} .
\end{aligned}
$$

Effects of the cyclotron self-absorption may be important for nonrelativistic electrons, where radiation itself is low. With increasing of the electron temperature self-absorption effects decrease, because the black-body emission increase with a temperature $\left(\sim T^{4}\right)$ more rapidly then magneto-Bremstrahlung volume losses. Account of self-absorption was investigated by Trubnikov (1973).

In the case of a disk accretion there are several characteristic velocities, $v_{\mathrm{K}}, v_{\mathrm{r}}, v_{\mathrm{s}}$, and $v_{\mathrm{t}}=\alpha v_{\mathrm{s}}$, all of which may be used for determining "equipartition" magnetic energy, and one characteristic length $h$. Consider three possible choices with $v_{\mathrm{K}}^{2}, v_{\mathrm{r}}^{2}$, and $v_{\mathrm{t}}^{2}$ for scaling $B^{2}$, note that choice of cross products of different velocities and of $v_{\mathrm{s}}$, is also possible. We get the following expressions for magnetic field strengths in different cases, with account of Eq. (68)

$$
\begin{aligned}
& \text { (a) } v_{\mathrm{B}}=v_{\mathrm{K}}, \quad \frac{B^{2}}{8 \pi}=\frac{\rho v_{\mathrm{K}}^{2}}{2}, \quad B=\left(\frac{\dot{M}}{\alpha \sqrt{2}} \frac{v_{\mathrm{K}}^{4} \mathscr{J}}{r^{2} v_{\mathrm{s}}^{3}}\right)^{1 / 2}, \\
& \text { (b) } v_{\mathrm{B}}=v_{\mathrm{r}}, \quad \frac{B^{2}}{8 \pi}=\frac{\rho v_{\mathrm{r}}^{2}}{2}, \quad B=\left(\frac{\dot{M} \alpha}{\sqrt{2} \mathscr{J}} \frac{v_{\mathrm{s}}}{r^{2}}\right)^{1 / 2}, \\
& \text { (c) } v_{\mathrm{B}} \sim v_{\mathrm{t}}, \quad \frac{B^{2}}{8 \pi}=\frac{\alpha_{\mathrm{m}}^{2}}{\alpha^{2}} \frac{\rho v_{\mathrm{t}}^{2}}{2}, \quad B=\alpha_{\mathrm{m}}\left(\frac{\dot{M}}{\alpha \sqrt{2}} \frac{v_{\mathrm{K}}^{2} \mathscr{J}}{r^{2} v_{\mathrm{s}}}\right)^{1 / 2} .
\end{aligned}
$$

The expression for an Ohmic heating in the turbulent accretion disc also may be written in different ways, using different velocities $v_{\mathrm{E}}$ in the expression for an effective electrical field

$$
\varepsilon=\frac{v_{\mathrm{E}} B}{c}
$$

A self-consistency of the model requires, that expressions for a magnetic heating of the matter $\mathscr{H}_{\mathrm{B}}$ obtained from the condition of stationarity of the flow Eq. (58), and from the Ohm's law Eq. (64), should be identical. That gives some restrictions for the choice of a characteristic velocity in Eq. (75). Comparison between Eqs. (58) and (64) with account of Eqs. (63) and (68) show the identity of these two expressions at

$$
v_{\mathrm{E}}=v_{\mathrm{r}}, \quad \frac{\alpha}{\mathscr{J} \alpha_{\mathrm{m}}}=\frac{3 \sqrt{2}}{4} .
$$

So, the model is becoming self-consistent at the reasonable choice of the parameters. Note, that in the advective models $\mathscr{J}$ is substituted by another function, which is not zero at the inner edge of the disc. The heating due to magnetic field reconnection $\mathscr{H}_{\mathrm{B}}$ in the Eqs. (65) and (66), obtained from Eqs. (58) and (74), may be written with account of Eq. (67) as

$$
\mathscr{H}_{\mathrm{B}}=\frac{3}{16 \pi} \frac{B^{2}}{r \rho} v_{\mathrm{r}}=\frac{1}{2 \mathscr{J}} \mathscr{H}_{\eta \mathrm{i}}\left(\frac{v_{\mathrm{B}}}{v_{\mathrm{K}}}\right)^{2} .
$$

So, at $v_{\mathrm{B}}=v_{\mathrm{K}}$ the expressions for viscous and magnetic heating are almost identical. The distribution of the magnetic heating between electrons and ions has a critical influence on the model, if we neglect the influence of a plasma turbulence on the energy relaxation, and take into account only the energy exchange by binary collisions from Eq. (69). Observations of the magnetic field reconnection in the solar flares show (Tsuneta, 1996), that electronic heating prevails.

It follows from the physical picture of the field reconnection, that transformation of the magnetic energy into a heat is connected with the change of the magnetic flux, generation of the vortex electrical field, 
accelerating the particles. This vortex field has a scale of the turbulent element and suffers rapid and chaotic changes. The accelerating forces on electrons and protons in this fields are identical, but accelerations themselves differ $\sim 2000$ times, so during a sufficiently short time of the turbulent pulsation the electron may gain much larger energy, then the protons. Additional particle acceleration and heating happens on the shock fronts, appearing around turbulent cells, where reconnection happens. In this process acceleration of the electrons is also more effective than of the protons. The Eqs. (65) and (66) have been solved (Bisnovatyi-Kogan and Lovelace, 1997) in the approximation of nonrelativistic electrons, $v_{\mathrm{B}=} v_{\mathrm{K}}$, what permitted to unite a viscous and magnetic heating into a unique formula. The combined heating of the electrons and ions were taken as

$$
\mathscr{H}_{\mathrm{e}}=(2-g) \mathscr{H}_{\eta \mathrm{i}}, \quad \mathscr{H}_{\mathrm{e}}=g \mathscr{H}_{\eta \mathrm{i}} .
$$

In the expression for a cyclotron emission selfabsorption was taken into account according to Trubnikov (1973). The results of calculations for $g=$ $0.5 \div 1$ show that almost all energy of the electrons is radiated, so the relative efficiency of the twotemperature, optically thin disc accretion cannot become lower then 0.25 . Note again that accurate account of a plasma turbulence for a thermal relaxation and corresponding increase of the term $Q_{\text {ie }}$ may restore the relative efficiency to its unity value, corresponding to the optically thick discs.

\section{DISCUSSION}

Observational evidences for existence of black holes inside our Galaxy and in the AGN (Cherepashchuk, 1996; Ho, 1999) make necessary to revise theoretical models of the disc accretion. Large part of high energy radiation indicates to its origin close to the black hole, where standard accretion disc model is not a appropriate. The improvements of a model are connected with account of advective terms and more accurate treatment of the magnetic field effects.
Conclusions about existence of ADAF solution for an optically thin accretion disc at low mass flux are connected with an incomplete account of the effects connected with magnetic field annihilation. Their account does not permit to make a relative efficiency of the accretion lower then $\sim 0.25$ from the standard value. It is expected that more accurate treatment of the relaxation connected with the plasma turbulence will even more increase the efficiency, making it close to unity (see also Fabian and Rees, 1995).

Some observational data which were interpreted as an evidence for the existence of the ADAF regime have disappeared after additional accumulation of data. The example of this sort is connected with the claim of the proof of the existence of event horizon of the black holes due to manifestation of the ADAF regime of accretion (Narayan et al., 1997). Analysis of the more complete set of the observational data (Chen et al., 1997) had shown disappearance of the statistical effect claimed as an evidence for ADAF. This example shows how dangerous is to base a proof of the theoretical model on the preliminary observational data, especially when the model is physically not fully consistent. Then even a reliable set of the observational data cannot serve as a proof of the model.

It looks like it is difficult to use ADAF for solution of the problem of existence of underluminous AGN, where the observed flux of the energy is smaller, then the expected from the standard accretion disc models. Two possible ways may be suggested. One is based on a more accurate estimation of the accretion mass flow into the black hole, which could be overestimated. Another, more attractive possibility, is based on existence of another mechanisms of the energy losses in the form of accelerated particles, like in the radiopulsars, where their losses exceed strongly a radiation losses. This is very probable to happen in a presence of a large scale magnetic field, which may be also responsible for a formation of the observed jets (Bisnovatyi-Kogan, 1999; Blandford and Begelman, 1999). To extend this line, we may suggest, that underlumilnous AGN loose main part of their energy to the formation of jets. The search of the correlation between existence of jets and lack of the luminosity could be very informative. 


\section{References}

Abramovicz, M.A., Czerny, B., Lasota, J.P. and Szuszkiewicz, E. (1988) ApJ 332, 646.

Artemova, I.V., Bisnovatyi-Kogan, G.S., Björnsson, G. and Novikov, I.D. (1996) ApJ 456, 119.

Balbus, S.A. and Hawley, J.F. (1998) Rev. Mod. Phys. 70, 1.

Bisnovatyi-Kogan, G.S. (1989) Physical Problems of the Theory of Stellar Evolution (Nauka, Moscow), in Russianin Russian.

Bisnovatyi-Kogan, G.S. (1993) A and A 274, 796.

Bisnovatyi-Kogan, G.S. (1999) In: Chakrabarti, S., ed, Observational Evidences for Black holes in the Universe (Kluwer, Dordrecht), $\mathrm{p} 1$.

Bisnovatyi-Kogan, G.S. and Blinnikov, S.I. (1976) Pisma Astron. Zh. 2, 489.

Bisnovatyi-Kogan, G.S. and Blinnikov, S.I. (1977) $A$ and A 59, 111. Bisnovatyi-Kogan, G.S. and Lovelace, R.V.L. (1997) ApJ 486, L43.

Bisnovatyi-Kogan, G.S. and Lovelace, R.V.L. (2000) ApJ 529, 978.

Bisnovatyi-Kogan, G.S. and Ruzmaikin, A.A. (1974) Astrophys. Space Sci. 28, 45 .

Bisnovatyi-Kogan, G.S. and Ruzmaikin, A.A. (1976) Astrophys. Space Sci. 42, 401.

Bisnovatyi-Kogan, G.S. and Sunyaev, R.A. (1972) Soviet Astron. 15, 697.

Blackman, E.G. (1997) astro-ph/9710137.

Blandford, R.D. and Begelman, M.C. (1999) MN R.A.S. 303, 1.

Bondi, H. (1952) MN R.A.S. 112, 195.

Bondi, H. and Hoyle, F. (1944) MN R.A.S. 104, 273.

Cannizzo, J.K., et al., (1998) AAS Abstracts 192, 4103C.

Chakrabarti, S.K. (1996) ApJ 464, 1996.

Chakrabarti, S.K. and Molteni, D. (1993) ApJ 417, 671.

Chang, K.M. and Ostriker, J.P. (1985) ApJ 288, 428.

Chen, W., Cui, W., Frank, J., King, A., Livio, M., Zhang, S.N. (1997) Talk on High Energy Ap. Division Meeting, November. Cherepashchuk, A.M. (1996) Uspekhi Fiz. Nauk 166, 809.

Di Matteo, T., Fabian, A.C., Rees, M.J., et al., (1999) Month. Not. R.A.S. 305, 492.
Fabian, A.C. and Rees, M.J. (1995) Month. Not. R.A.S. 277, L55.

Giacconi, R., Gursky, H., Paolini, F.R. and Rossi, B.B. (1962) Phys Rev. Lett. 9, 439.

Ho, L. (1999) In: Chakrabarti, S., ed, Observational Evidences for Black Holes in the Universe (Kluwer, Dordrecht), p 157.

Hõshi, R. and Shibazaki, N. (1977) Prog. Theor. Phys 58, 1759.

Ichimaru, S. (1977) ApJ 214, 830.

Landau, L.D. (1937) Zh. Exp. Theor. Phys. 7, 203.

Liang, E.P.T. and Thompson, K.A. (1980) ApJ 240, 271.

Lynden-Bell, D. (1969) Nature 223, 690.

Matsumoto, R., Sato, Sh., Fukue, J. and Okazaki, A.T. (1984) Publ. Astron. Soc. Japan 36, 71.

Menon, Quataert, E., Narayan, R. (1997) astro-ph/9712015.

Narayan, R. and Yu, I. (1995) ApJ 452, 710.

Narayan, R., Barret, D., McClintock, J.E. (1997a) astro$\mathrm{ph} / 9610014$.

Narayan, R., Garcia, M.R., McClintock, J.E. (1997b) astro$\mathrm{ph} / 9701139$.

Novikov, I.D. and Thorne, K.S. (1973) In: DeWitt, C. and DeWitt, B., eds, Black Holes (Gordon and Breach, New York), p 345.

Paczyńsci, B. and Bisnovatyi-Kogan, G.S. (1981) Acta Astron. 31, 283.

Paczyńsci, B. and Wiita, P.J. (1980) $A$ and A 88, 23.

Pringle, J.E. and Rees, M.J. (1972) $A$ and $A$ 21, 1.

Quataert, E. (1997) astro-ph/9710127.

Riffert, H. and Herold, H. (1995) ApJ 450, 508.

Schmidt, M. (1963) ApJ 136, 164.

Schwartsman, V.F. (1971) Soviet Astron. 15, 377.

Shakura, N.I. (1972) Astron. Zh. 49, 921.

Shakura, N.I. and Sunyaev, R.A. (1973) $A$ and A 24, 337.

Shapiro, S.L., Lightman, A.P. and Eardley, D.M. (1976) ApJ 204 187.

Spitzer, L. (1940) MNRAS 100, 396.

Trubnikov, B.A. (1973) Voprosy Teorii Plasmy 7, 274.

Tsuneta, S. (1996) ApJ 456, 840.

Velikhov, E.P. (1959) Sov. Phys. - JETP 36, 995. 


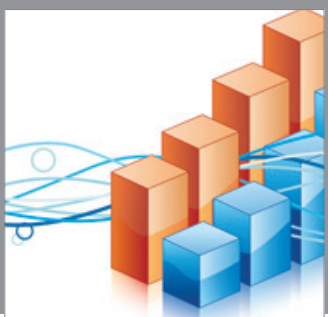

Advances in

Operations Research

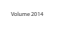

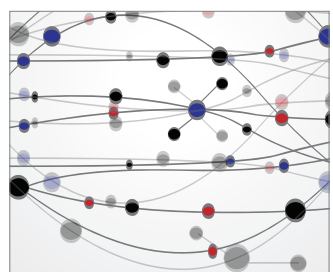

\section{The Scientific} World Journal
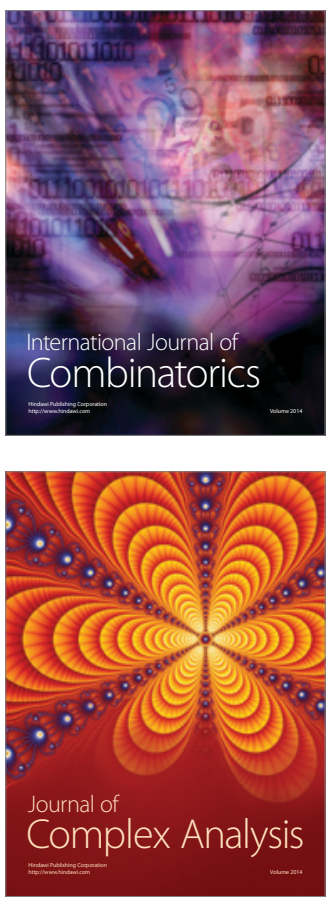

International Journal of

Mathematics and

Mathematical

Sciences
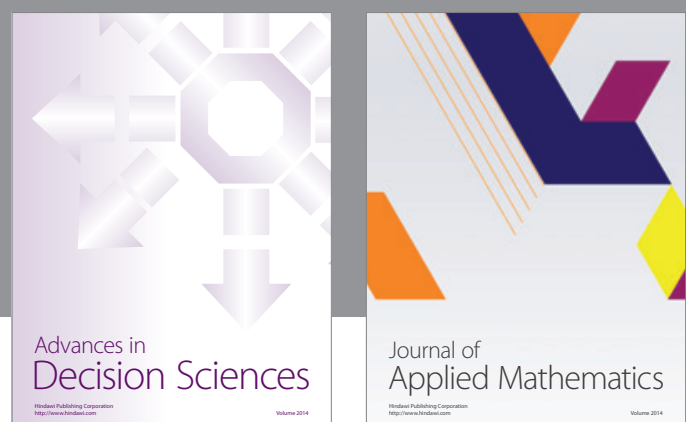

Journal of

Applied Mathematics
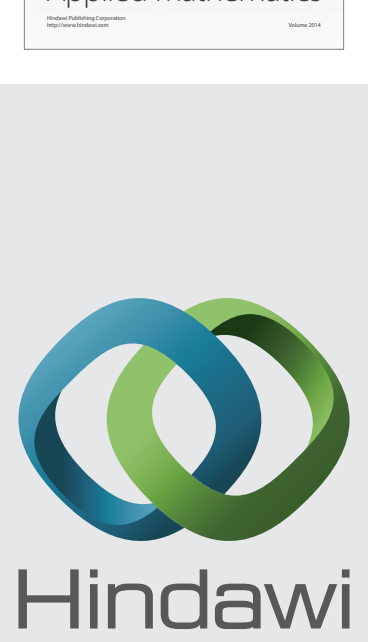

Submit your manuscripts at http://www.hindawi.com
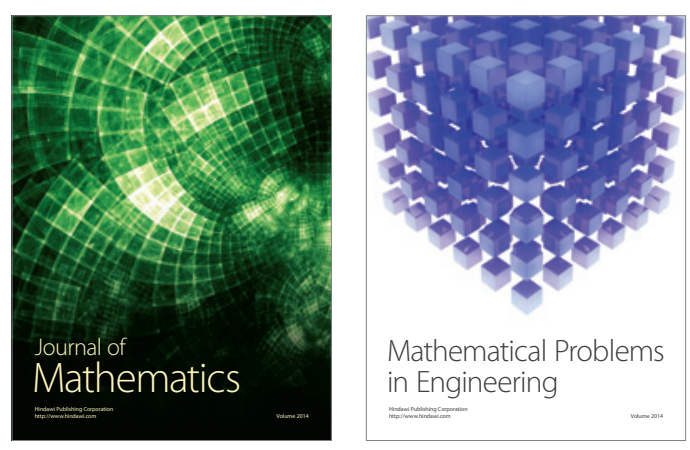

Mathematical Problems in Engineering
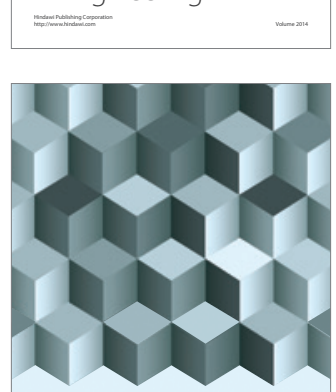

Journal of

Function Spaces
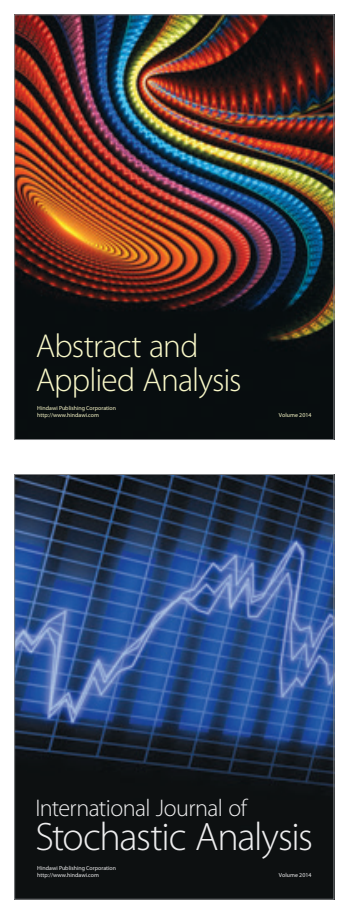

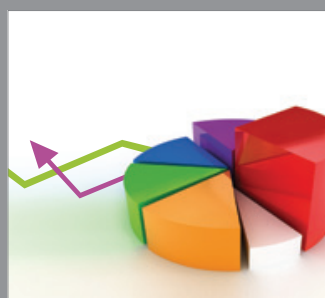

ournal of

Probability and Statistics

Promensencen
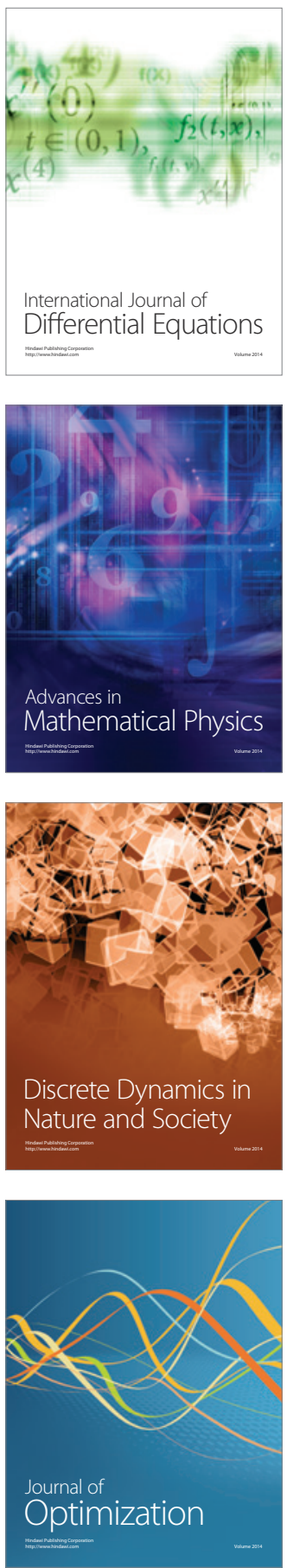